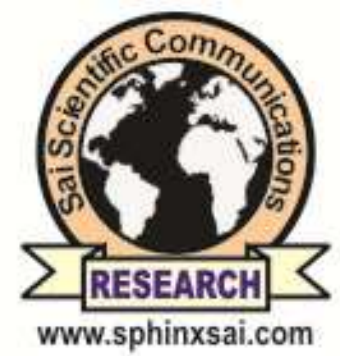

\title{
Subcorneal Pustular Dermatosis (Sneddon-Wilkinson Disease): A Rare Case
}

\author{
Rina Gustia $^{1^{\star}}$, Yosse Rizal ${ }^{2}$, Dwi Sepfourteen ${ }^{3}$ \\ ${ }^{1}$ Department of Dermatology-Venereology, Head of Non Infection Division, Dr. M. \\ Djamil Hospital, Medical Faculty of Andalas University, Padang, West Sumatera, \\ Indonesia \\ ${ }^{2}$ Department of Dermatology-Venereology, Achmad Mochtar Hospital, Medical Faculty \\ of Andalas University, Padang, West Sumatera, Indonesia \\ ${ }^{3}$ Department of Dermatology-Venereology, School of Medicine, Medical Faculty of \\ Andalas University, Padang, West Sumatera, Indonesia
}

\begin{abstract}
Subcorneal pustular dermatosis (SPD) is a rare, chronic, recurrent, pustular eruption. It may be difficult to differentiate SPD from Acute Generalized Exanthematous Pustulosis (AGEP) and Generalized Pustular Psoriasis (GPP)just from clinical appeareance. A 48 years old woman with subcorneal pustular dermatosis was reported. She was complained there were tiny blisters containing pus on the reddish patches that felt burning and became widespread to neck, chest, abdomen, arms, groin and legs since 4 days ago. Patient had no history of skin lesion before. From physical examination revealed no other pathologies and on the skin showed erythematous patches with annular lesion, pustules formation that tended to coalesce to form desquamative plaques, erosions, on chest, abdomen, back, arm, buttock and leg. Based on histopathology showed there are subcorneal pustules. The subcorneal pustule is filled with neutrophils and on the dermis, there are mild infiltration of neutrophil cells. This feature revealed to SPD.Clinically it may be difficult to differentiate SPD from AGEP and GPP. Histopathology examination can distinguished these disease,patient was treated with prednisone $40 \mathrm{mg}$ and dapson 100 $\mathrm{mg}$ and had improvement after 4 weeks.

Keywords : subcorneal pustular dermatosis, differential diagnosis, histopathologycal examination.
\end{abstract}

\section{Introduction}

Subcorneal pustular dermatosis (SPD) is a rare, chronic, recurrent, pustular eruption characterized histopathologically by subcorneal pustules that contain abundant neutrophils. The condition was originally described in 1956 by Sneddon and Wilkinson, who separated SPD from other previously unclassified pustular eruptions. The incidence of SPD is unknown. More than 200 cases were reported in a review published in 1981 (Chimenti and Ackerman, 1981). Racial predilection and geographic distribution have not been reported. SPD is more common in females than in males (ratio 4:1) and has been rarely reported in children. SPD usually presents between the fifth and seventh decades of life, but SPD may occur at any age. ${ }^{1,2}$

Rina Gustia et al /International Journal of PharmTech Research, 2020,13(4): 309-315.

DOI: http://dx.doi.org/10.20902/IJPTR.2019.130401 
There is still no clear evidence about the cause of SPD. The trigger such as concomitant infections, though repeatedly discussed, has remained speculative. Cases in association with pyoderma gangrenosum, benign monoclonal IgA gammopathy and multiple myeloma are well documented. There are also anecdotal reports in association with mycoplasma pneumoniae respiratory infection,seronegative and seropositive rheumatoid arthritis,hypothyroidism and hyperthyroidism, systemic lupus erythematosis, Sjogren's syndromeCrohn's disease, multiple sclerosisand SAPHO (synovitis, acne, pustulosis, hyperostosis, osteitis) syndrome. ${ }^{1,3}$

The primary lesions of SPD are small, discrete, flaccid pustules, or vesicles that rapidly turn pustular and usually arise in crops within a few hours on clinically normal or slightly erythematous skin. In dependent regions, pus characteristically accumulates in the lower half of the pustule; as the pustules usually have the tendency to coalesce, they often, but not always, form annular, circinate, or bizarre serpiginous patterns. After a few days, the pustules rupture and dry up to form thin, superficial scales and crusts, closely resembling impetigo. Peripheral spreading and central healing leave polycyclic, erythematous areas in which new pustules arise as others disappear. There is no atrophy or scarring, but an occasional brownish hyperpigmentation may mark previously affected sites. Variable intervals of quiescence, lasting from a few days to several weeks, may be followed by the sudden development of new lesions. The eruptions tend to occur symmetrically, affecting mainly the axillae, groin, abdomen, submammary areas, and the flexor aspects of the limbs. In rare cases, the face,palms, and soles may be involved. Scalp and mucous membranes invariably remain free of lesions. Episodic itching and burning represent subjective symptoms in a small number of patients, but there are no systemic symptoms or abnormalities in routine laboratory parameters. ${ }^{1,4}$

Histological feature of SPD consist with accumulation of neutrophils, absence of spongiosis or acantholysis.In classic SPD, direct and indirect immunofluorescence studies are negative. Cultures of the pustules consistently do not reveal bacterial growth. ${ }^{1}$

The main differential diagnoses of SPD include pustular psoriasis, the subcorneal type of IgA pemphigus, pemphigus foliaceus, dermatitis herpetiformis, impetigo and acute generalized exanthematous pustulosis. ${ }^{1}$

The drug of choice for SPD is dapsone at doses of 50-150 mg daily. Resolution is in about 4 weeks, but in some cases it may be refractory, and toxicity such as methaemoglobinaemia or haemolytic anaemia may be a limiting factor. Most cases require long-term dapsone to maintain disease control. Systemic steroid and retinoid acid are alternatives when dapsone can not be used. Therapeutic alternatives include retinoids, phototreatment with psoralen ultraviolet (UV) A, broadband or narrow band UVB and corticosteroids. ${ }^{1,2,5}$

\section{Case Report}

A 48 year old woman with tiny blisters containing pus on the reddish patches with burning sensation and spread along the chest, abdomen, back, arms, buttock and legs since 3 month ago. Patient had no history of skin lesion before. Physical examination revealed no other pathologies and on the skin showed erythematous patches with annular lesion, pustules formation that tended to coalesce to form desquamative plaques, erosions, on chest, abdomen, back, arm, buttock and leg (Figure.1). Results of laboratory examinations including complete blood count, serum chemistries, serum protein electrophoresis, urine protein electrophoresis, and glucose-6-phosphate dehydrogenase (G6PD) were normal. Cultures of the pustules were sterile.The differential diagnosis included AGEP and Generalized pustular psoriasis. Histopathology examination showed there was skin tissue with epidermis consists of stratified squamous epithelium, and on some places there are subcorneal pustules. The subcorneal pustule is filled with neutrophils and on the dermis, there are mild infiltration of neutrophil cells. This feature revealed to SPD.The patient was treated with prednisone $40 \mathrm{mg}$, dapsone $100 \mathrm{mg}$, ranitidine $2 \times 150 \mathrm{mg}$ and topical corticosteroids (mometason furoat ointment $0,1 \%$ on erythematous patches on trunk and both of arm and clobetasol ointment for erythematous patches on the leg). Oral corticosteroid was given during the first days hospitalization because she was suspected with AGEP. After histopathological examination pointed out the characteristic of SPD, dapsone was administered orally $100 \mathrm{mg} /$ day.Prednisone was tapered off after 4 weeks and dapsone still continued $100 \mathrm{mg} /$ day. Complete remission of the dermatosis was achieved after got dapsone for five months. During seventh months of follow-up, no relapse of the disease has occurred. 


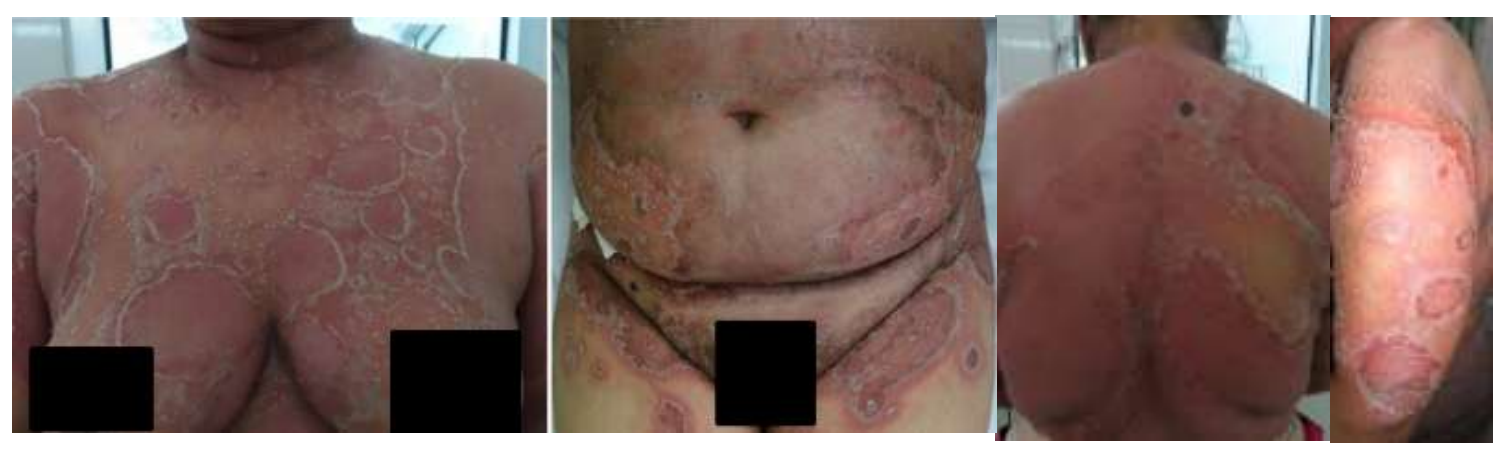

Figure 1. Annular, circinate erythematous plaque with pustules, yellowish squama on the chest. B. Annular, circinate erythematous plaque with pustules, yellowish squama on the abdomen, pubic and both of inguinal. C. Annular, circinate erythematous plaque with pustules, yellowish squama on the back. D. Annular, circinate erythematous plaque with pustules, yellowish scale on the both of arm. (yellow arrow : annular lesion)

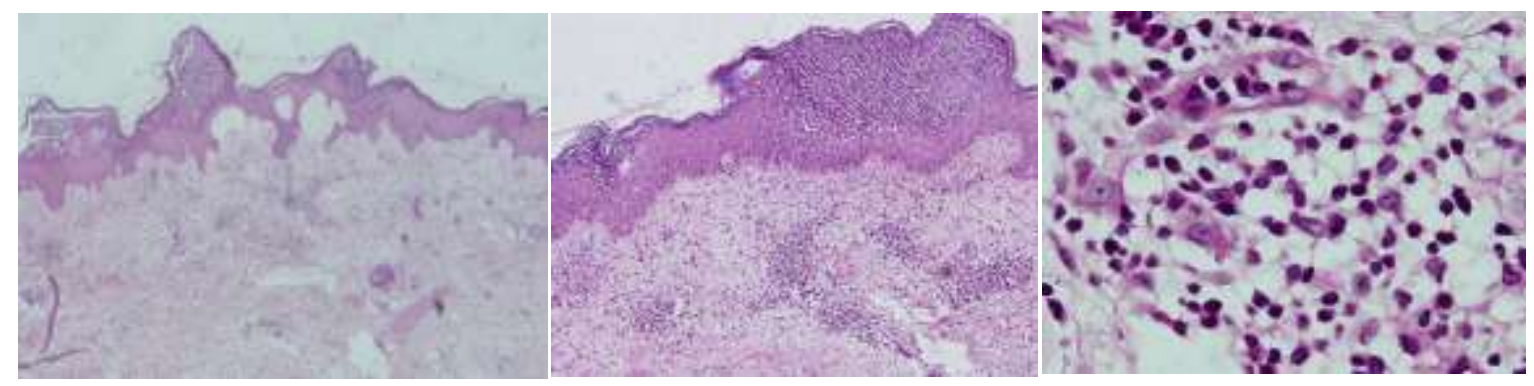

Figure 2. A.Epidermis consists of stratified squamous epithelium, and on some places there are subcorneal pustules. HE stain. enlargement $10 \mathrm{X}$ and $4 \mathrm{x10}$. B. Subcorneal pustule, consists of neutrophils, enlargement 20x10. C. There are Acantholysis. Enlargement 4 x 10.
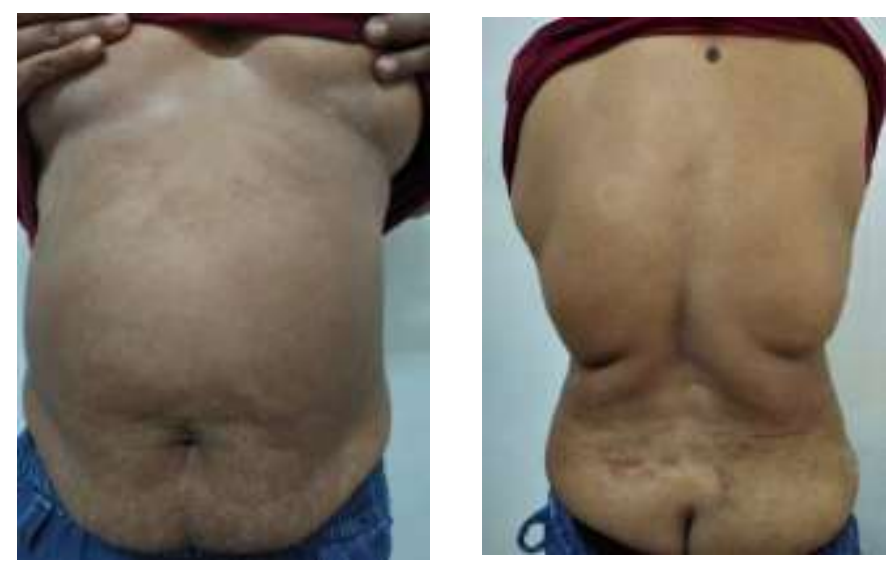

Figure 3. After four weeks got a treatment with prednisone $40 \mathrm{mg}$ and dapsone $100 \mathrm{mg}$. A. healed with hyperpigmentation.B.back, heal withhyperpigmentation patches. 


\section{Discussion}

Subcorneal Pustular Dermatosis should be differentiated from a wide spectrum of skin diseases that progress with pustular eruption, such as bacterial folliculitis, acne, localized pustular contact dermatitis, Sweet syndrome, impetigo, pemphigus foliaceus, staphylococcal scalded skin syndrome, subcorneal pustular dermatosis, and generalized pustular psoriasis (GPP). ${ }^{1}$ We reported of a case of subcorneal pustular dermatosis and make differential diagnosis with AGEP and generalized pustular psoriasis (GPP).To distinguished them required histopathological examination. ${ }^{1}$

Subcorneal pustular dermatosis progresses with subacute development of larger pustules, often arranged in a circinate pattern. The clinical recurrence of similar rash and longer periods of lesions and high fever should suggest GPP. Acute generalized exanthematous pustulosis is is characterized by sudden onset of an eruption of numerous, small $(<5 \mathrm{~mm})$, non-follicular pustules, high fever $\left(>38^{\circ} \mathrm{C}\right)$, neutrophilia, and acute evolution with spontaneous resolution in less than 15 days. Other rare skin signs are edema of the face, purpura, atypical target-like lesions, and vesicles. ${ }^{1,5,6}$
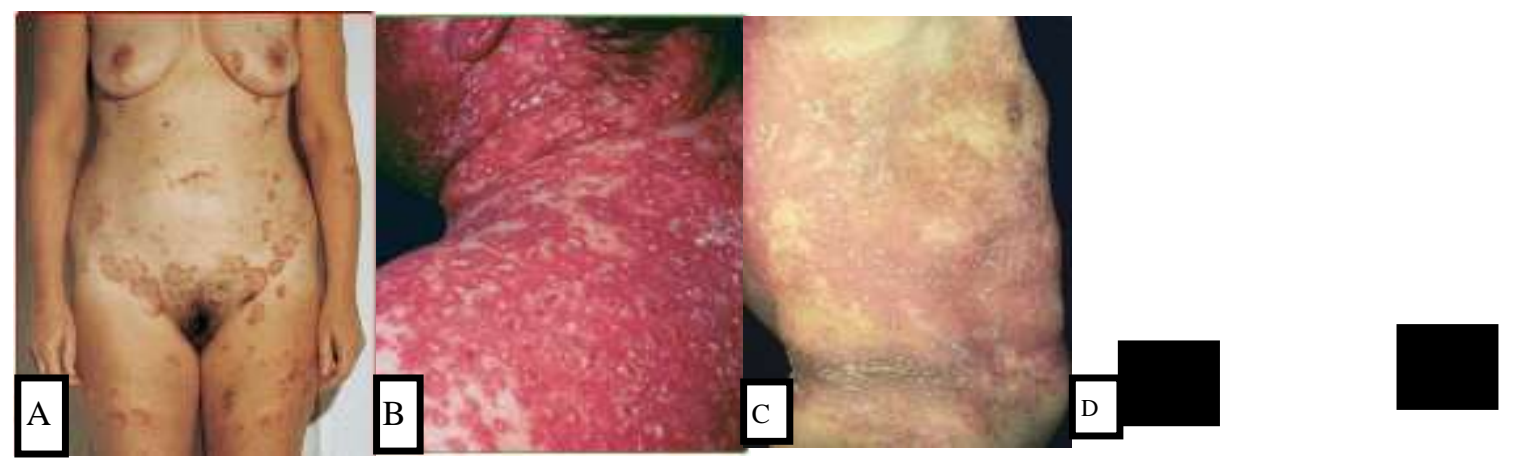

Figure 4. A. Subcorneal pustular dermatosis. B. Acute exanthematous generalized dermatosis. C. generalized pustular psoriasis. D.In this case, Subcorneal pustular dermatosis. Fig. A-C. From references no.1

Although differentiation of SPD from AGEP, GPP may be difficult, it can be differentiated by histopathological analysis. Histopathologically, SPD characterized by subcorneal pustules that contain abundant neutrophils with the absence of spongiosis or acantholysis, although acantolysis may be reported in older lesions. Neutrophils migrate through the epidermis to aggregate beneath the stratum corneum, leading to sterile subcorneal pustules. While widespread edema on the papillary dermis, vasculitis, perivascular eosinophil infiltration, and/or focal keratinocyte necrosis favor AGEP. Kogoj's spongiform pustule has been regarded as pathognomonic finding in pustular psoriasis. It's shows neutrophil accumulation beneath the stratum corneum and swollen or even damaged keratinocytes in addition to those histological findings seen in skin lession of psoriasis vulgaris including parakeratotic hyperkeratosis, Munro's microabscess, elongation of rete ridges, capillary dilatation in the edematous papillary dermis, and a superfiicial perivascular infiltration of mononuclear cells in the upper dermis.Subcorneal pustular dermatosis progresses with subacute development of larger pustules, often arranged in a circinate pattern. ${ }^{7,8,9}$

Histopathology examination result in this case showed bullae containing lymphocytes cells, polymorphonuclear leukocyte cells, flattened epithelial cells and debris on sub corneal layer, there are acantholysis and on the dermis, there are blood vessels with hyperemia and classify cell infiltration of lymphocytes, plasma cells, polymorphonuclear leukocytes cells, and some eosinophils. In this case consistent with SPD. 

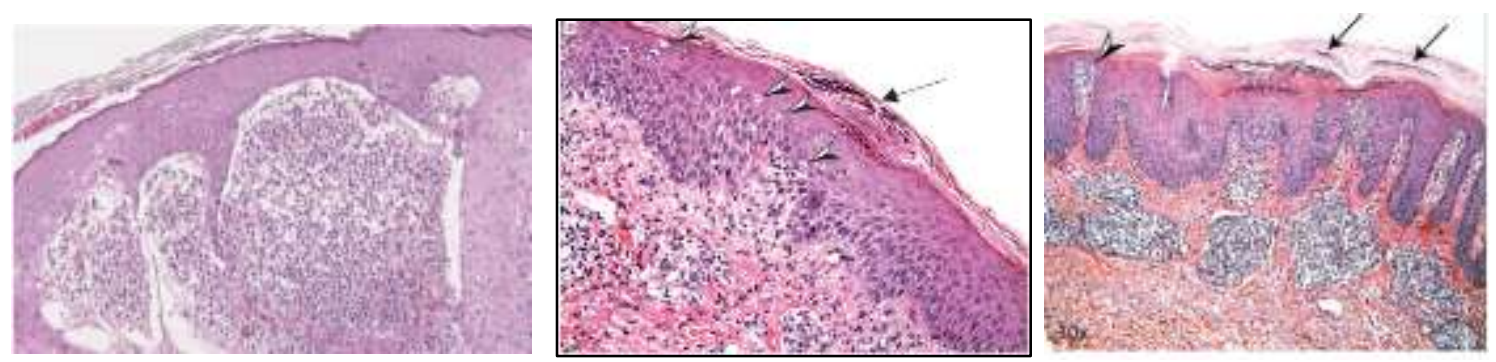

Figure 5.A. Subcorneal pustular dermatosis, subcorneal pustule below the stratum corneum containing mainly neutrophils. (Ref. no.9)B. Histopathology of AGEP, small sub-/intracorneal pustule contiguous with a Munro-like abscess (arrow), spongiosis, few epidermal necrotic keratinocytes (arrowheads), erythrocyte extravasation, discrete leukocytoclasia and mixed perivascular and interstitial infiltrate including eosinophils.(Ref. no.7).C. Histopathology ofchronic generalized pustular psoriasis; Club-shaped psoriatic rete ridges with hyperkeratosis, parakeratosis, Munro abscesses (arrow), epidermal plate thinning and sub/intracorneal pustule with dilated, tortiou vessels (arrowhead) and superficial perivascular mononuclear infiltrates (ref. no.5).

Several therapeutic options have been proposed for the treatment of SPD.Dapsone (50-150 mg/day), the main agent in SPD, inhibits the cytotoxic effects of peripheral neutrophils. A response to dapsone is typical of SPD and has been used to suggest a diagnosis of SPD. ${ }^{10}$ The response to dapsone is slower andless dramatic than in dermatitis herpetiformis, butcomplete remission is most often obtained. In somepatients, the treatment may be withdrawn afterseveral months, although in others it may have to becontinued for years; the minimal effective dose to suppressdisease should be determined in these patientswith a chronic course. ${ }^{1}$ Oral corticosteroids are generally ineffective,but their combination with dapsone seems to be useful. Topical corticosteroids have been used alone or in addition to dapsone. ${ }^{11}$ In this case, patient got prednisone $40 \mathrm{mg}$, dapsone $100 \mathrm{mg} / \mathrm{day}$ and topical corticosteroid. Prednisone tapered after four week. Complete remission after five month.During seventh months of follow-up, no relapse of the disease has occurred.

\section{Conclusion:}

Subcorneal Pustular Dermatosisis an entity that often becomes chronic.It may be difficult to differentiate SPD from AGEP and GPP.The terms support to SPD: patient is a woman, age > 40 years old, the pustules initially appear on SPD's predilection, there were flaccid pustules that coalesce leaving a desquamative plaques, culture and sensitivity from pus was steril, and there were no fever and leucocytosis.Histopathology examination is needed to confirmed a diagnosis of SPD. Patient was treated with combination prednisone $40 \mathrm{mg}$ and dapsone $100 \mathrm{mg} /$ day, showed good result after five months and no reccurence of the disease has occurred during seventh months follow up.

\section{References}

1. Trautinger F, Hönigsmann H. Subcorneal pustular dermatosis (Sneddon-Wilkinson disease). In: Kang S et al., editors. Fitzspatrick's dermatology in general medicine. 9th ed. united state of America; 2019. p. 617-20.

2. Bhargava S, Kumar U, Kroumpouzos G. Subcorneal pustular dermatosis:Comprehensive review and report of a case presenting during pregnancy. Int J Women's Dermatology [Internet]. 2020;6(3):131-6.

3. Bohelay G, Duong TA, Ortonne N, Chosidow O, Valeyrie-Allanore L. Subcorneal pustular dermatosis triggered by Mycoplasma pneumoniae infection: A rare clinical association. J Eur Acad Dermatology Venereol. 2015;29(5):1022-5.

4. Şahin A, Edgüer EY, Astarci HM, Ekşioğlu HM. Subkorneal püstüler dermatoz "Sneddon-Wilkinson hastaliği": Bir olgu sunumu. Turkderm Deri Hast ve Frengi Ars. 2017;51(1):21-3.

5. Momin SB, Del Rosso JQ, Michaels B, Mobini N. Acute generalized exanthematous pustulosis: An enigmatic drug-induced reaction. Cutis. 2009;83(6):291-8. 
6. Vougas V, Dedemadi G, Noutsis K, Th A, Pantelidaki C, Drakopoulos S. Generalised Pustular Psoriasis ( von Zumbusch type ) following renal Transplantation . Report of a case and review of the literature. 2007;2(2):89-93.

7. Kardaun SH, Kuiper H, Fidler V, Jonkman MF. The histopathological spectrum of acute generalized exanthematous pustulosis (AGEP) and its differentiation from generalized pustular psoriasis. J Cutan Pathol. 2010;37(12):1220-9.

8. Abreu-velez AM, Smith JG, Howard MS. Case Report Subcorneal pustular dermatosis an immnohistopathological perspective. 2011;4(5):526-9.

9. Ferrillo M, Villani A, Fabbrocini G, Mascolo M, Megna M, Costa C, et al. A case of the co-existence of subcorneal pustular dermatosis and pyoderma gangrenosum and a review of the literature. Open Access Maced J Med Sci. 2018;6(7):1271-4.

10. Cheng S, Edmonds E, Ben-Gashir M, Yu RC. Subcorneal pustular dermatosis: 50 Years on. Clin Exp Dermatol. 2008;33(3):229-33.

11. Bordignon M, Zattra E, Montesco MC, Alaibac M. ( Sneddon-Wilkinson Disease ) with Absence of Desmoglein 1 and 3 Antibodies Case Report and Literature Review. Am J Clin Derm. 2008;9(1):51-5. 
For your Research References Requirements, Always log on to www.sphinxsai.com

\section{International Journal of PharmTech Research \\ International Journal of ChemTech Research}

